\title{
An qudit on the reporting of dental caries on radiographs
}

\section{By Govind Malhi ${ }^{1}$}

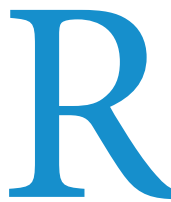

adiographs are a valuable tool for dentists and other dental care professionals (DCPs) for a variety of reasons: including diagnosing caries to assessing bone loss in periodontal disease. Radiographs can be extremely useful during the treatment of a patient by a dental professional. The use of radiographs, most notably bitewings, in the detection of caries (especially in children) cannot be understated. According to the Faculty of General Dental Practice (FGDP), 'posterior bitewings are an essential adjunct to clinical examination.' ${ }^{1}$ Such a statement highlights the importance of radiographs in caries detection and therefore the importance of good subsequent reporting and assessment of the disease.

It is for this reason that caries reporting on dental radiographs was chosen as this audit topic. The aims of the audit are to monitor and assess the presence and quality of caries reporting on bitewings and periapical radiographs taken during dental foundation training. Newly qualified dentists can often feel the pressure of time constraints in general practice compared to patient clinics in university, where they are afforded much more time for clinical exams and radiographic reporting. As a result, the quality of radiograph

\section{Author information}

'Dr Govind Malhi BDS, DCT1 in Oral and Maxillofacial Surgery, Royal Cornwall Hospital reports can be overlooked when attempting to complete a full clinical exam in acceptable time (ie before the next patient comes in).

The objective of this audit was to gain $100 \%$ completion of a radiographic report with a dedicated section on reporting on caries. One hundred percent was chosen as the standard due to the importance of detecting caries by dental professionals. Studies have shown that the median time for caries penetrating the enamel to form is 6.1 months. ${ }^{2}$ This highlights the need for regular radiographic assessment of dental caries if an individual is deemed high caries risk.

\section{Method}

A total of 25 bitewing radiographs and 25 periapical radiographs were assessed for a report on the presence of caries on the radiograph. Table 1 was used to collate the information.

A caries report was classed as an obvious description of whether caries was detected in the radiograph. If a caries report was evident then a 'yes' was placed in the table. If there was no caries present then a 'no' was placed in the table. In some instances, there was the possibility that a caries assessment was done however, if there was no caries present on the radiograph, it was not written in the radiographic report. This would subsequently result in a 'no' being placed in the table.

The actual diagnosis of the carious lesions was done with the aid of Fejerskov et al. ${ }^{3}$

\section{Results}

The results of the first audit cycle are shown in Figure 1.

From this graph we can see that we have fallen far below the standard set for both bitewing radiographs and periapical radiographs. As mentioned above, the most likely reason for this is the lack of reporting on caries if none were detected in the radiographs.
However, this can be a detrimental habit to implement into the practice of a dental professional as a lack of a report on caries can potentially result in legal issues for that individual, even if no caries was present.

\section{Action plan}

It was clear that adjustments had to be made in the reporting of caries in a radiographic report. An adjustment had to be made that was effective and ensured that the lack of reporting on dental caries on a radiograph was eliminated. The action plan chosen was a template to be used on the EXACT dental software (the software used during this audit) which will ensure a caries report was written each time a radiograph report was done. The template used is shown below:

Type of radiograph:

Justification:

Dose (mSv):

Image quality (grade):

\section{Caries:}

Bone Levels:

Other findings:

The reason why a software template was chosen was because if the template was used but the section dedicated to caries was not filled in, the notes would be regarded as incomplete, which, as mentioned above, could have legal ramifications for the dental professional.

\section{Results 2}

The results of the second cycle are shown in Figure 2.

As we can see from this graph, a $100 \%$ success rate of a caries report written in a radiograph report was met meaning the standard of $100 \%$ success was met. This 
highlights the effectiveness of the action plan that was implemented after the first cycle. The use of the template ensured that a full radiograph report was completed with a dedicated section for caries included.

\section{Conclusion and recommendations}

In conclusion, it can be said that this audit cycle was a success which is a very rewarding feeling. This audit not only resulted in a better record keeping system, it resulted in an improvement in dental practice, which is extremely important during the early years of a dental career.

This audit also allowed two General Dental Council (GDC) standards to be met: Standard 4 - Maintain and protect patients information (specifically 4.1.2 which mentions recording as much detail as possible) and Standard 7 - Maintain, develop and work within our professional knowledge and skills (specifically 7.3.2 which mentions partaking in activities that develop your knowledge and skills). ${ }^{4}$ Being able to meet and uphold two of the standards that all dental professionals abide by is very fulfilling.

In terms of future recommendations, it should be recommended that all dental professionals that use the EXACT dental software (and any other software that allows note templates to be used) should consider implementing the template used in this audit into their general practice. The audit showed how effective a simple template can be in improving the quality of a radiographic report. Therefore, if more dental professionals adopt the use of the template, the overall quality of radiographic reports will improve which has the potential to reduce litigation claims against dental professionals. This has significance as a survey carried out by Dental Protection found almost $90 \%$ of dentists have fears of being sued. The survey also found that $74 \%$ of dentists felt that this fear was affecting the services they were able to offer. ${ }^{5}$ As a result, it can be said that the use of a template to improve the quality of a radiograph report can not only improve the general practice of a dental professional but could also result in a possible improvement in mental health, as better radiograph record keeping will reduce the likelihood of a claim being escalated.

After seeing the success of this first audit, it is hoped that a practice-wide audit can be carried out. By monitoring and improving the quality of radiographic reports by other dental professionals, the practice as a whole will have the potential to improve in not only record keeping, but also overall patient care.

\section{References}

1. FGDP(UK). 4.2 Children. In: Selection criteria for dental radiography. Updated

Table 1 This table shows how the information for the audit was collated (this table is an example of the one used.The real table was extended to reflect the number of radiographs assessed)

\begin{tabular}{|l|l|l|l|l|l|l|}
\hline Caries report written & $1^{\text {st }}$ Cycle \\
\hline 2nd Cycle & $2^{\text {nd }}$ \\
\hline
\end{tabular}
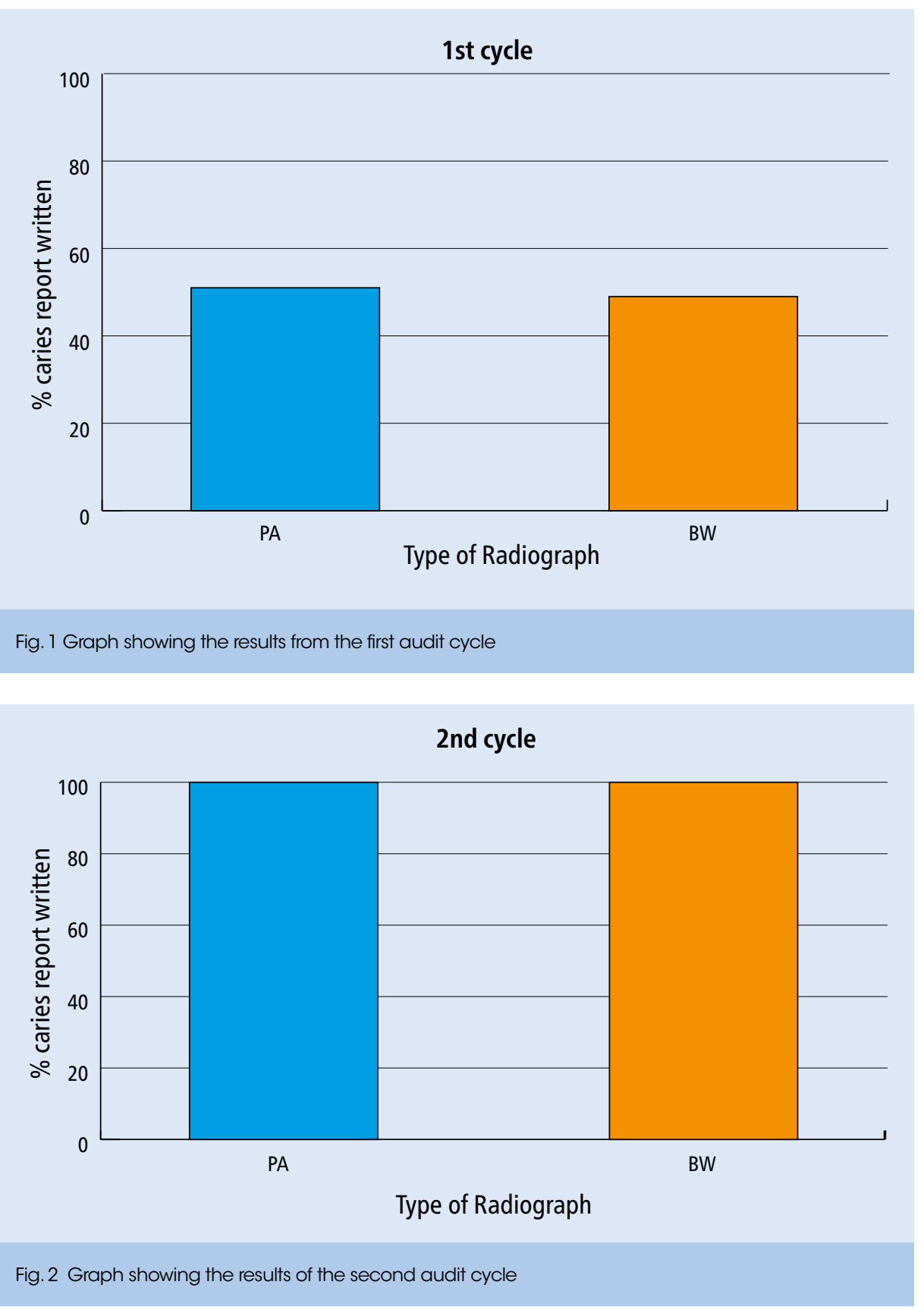

February 2018. Available at: https://www. fgdp.org.uk/selection-criteria-dentalradiography/42-children (accessed 9 March 2021).

2. Arrow P. Incidence and progression of approximal carious lesions among school children in Western Australia. Aust Dent J 2007; 52: 216-226.

3. Fejerskov O, Nyvad B, Kidd E (eds).
Radiography for caries diagnosis. In: Dental caries: the disease and its clinical management, 2nd edition. p 72. Blackwell, 2008.

4. General Dental Council. Standards for dental professionals, 2017.

5. BDJ News. Almost $90 \%$ of dentists have fears of being sued by patients. $\mathrm{Br}$ Dent $J$ 2018; 225: 798. 